\title{
A SYMMETRIC DEFORMATION-BASED SIMILARITY MEASURE FOR SHAPE ANALYSIS
}

\author{
S. Kolouri ${ }^{1}$, D. Slepčev ${ }^{2}$, G. K. Rohde $e^{1,3}$ \\ ${ }^{1}$ Center for Bioimage Informatics, Biomedical Engineering Department \\ ${ }^{2}$ Department of Mathematical Sciences \\ ${ }^{3}$ Lane Center for Computational Biology \\ Carnegie Mellon University, Pittsburgh, PA, 15213.
}

\begin{abstract}
Statistical modeling of cellular/subcellular shapes is required for quantifying shape variations and understanding complex cell behaviors. Such statistical models rely on the choice of a proper similarity measure. In this paper we introduce a symmetric deformation-based similarity measure for cellular shape analysis. The proposed method is based on finding an optimal diffeomorphic mapping between shape images that minimizes a physically meaningful energy function. We compare our proposed method to the large deformation diffeomorphic metric mapping (LDDMM) and the standard Euclidean metric on a nuclei dataset and show that the proposed method outperforms the others in capturing the variations in the dataset.
\end{abstract}

Index Terms - Shape analysis, image registration, machine learning

\section{INTRODUCTION}

Statistical modeling of shape variations is a very important task in a plethora of disciplines, including brain research, phylogenetic research, plant taxonomy, cell biology, archaeology, etc $[1,2,3]$. In cell biology, for instance, statistical modeling of cell shapes provides quantitative information, which can be used to distinguish normal and diseased cells [3].

A proper statistical model for shapes requires a physically meaningful similarity measure to capture variations [4]. Conventionally, descriptors are used to represent the shape space and the similarity measure is defined as the Euclidean distance between these descriptors [5]. Area, perimeter, eccentricity, boundary curvature, thinness, equivalent diameter, etc. are some common shape descriptors that are frequently used in the literature. Such statistical modeling usually induces a precise model of the shape space, but it suffers from several shortcomings. Most importantly, such shape space is not generative. Meaning that, a transform from the image space to the descriptor space is generally non-invertible [3]. Contour-based shape representation, point distribution, and active shapes are other models used for shape analysis

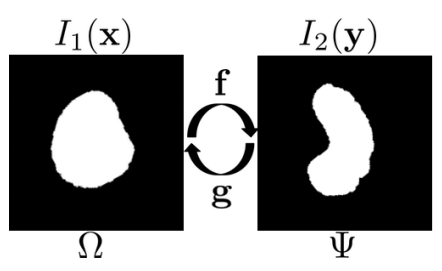

Fig. 1. Two shape images of HeLa cell nuclei, $I_{1}$ and $I_{2}$ with their corresponding domains, $\Omega$ and $\Psi$, and mappings f : $\Omega \rightarrow \Psi$ and $\mathbf{g}: \Psi \rightarrow \Omega$.

$[6,7,8]$. Contour-based techniques are suitable for modeling shape variations, however they lack information about the internal changes of the shape.

Here, our focus is on deformation-based shape analysis which is an alternative way of constructing a statistical model for shapes. In such methods, one shape is deformed to another and the statistical model is obtained from the deformation mappings. In addition, the similarity measure is defined as a functional of the deformation mapping. Rohde et al. [3], for instance, proposed a deformation-based shape analysis method using the large deformation diffeomorphic metric mapping (LDDMM) [9] framework in combination with the multidimensional scaling (MDS) [10].

In this paper, we propose a symmetric deformation-based similarity measure (SDBM) technique with application to cell/nuclei shape analysis. The proposed technique is based on a multi-resolution non-linear registration scheme, and measures physically meaningful variations among the shapes. Our method provides an efficient numerical scheme for finding the deformation-based similarities among shapes.

\section{METHOD}

Deformation-based methods [9, 11] commonly represent the deformations as end points of paths of diffeomorphisms parametrized with a smooth time-varying 'velocity' fields. Although mathematically elegant, such methods are computationally expensive, as they require time varying deformation 
fields. This makes such methods unsuitable for deformationbased pattern recognition on large datasets. Our approach, on the other hand, relaxes the problem by finding a time independent deformation field. Our proposed method can be thought of as a linearization of the other problem.

A set of homeomorphic shapes can be related by a group of diffeomorphisms, which are one to one smooth differentiable and invertible mappings. Let $I_{1}: \Omega \rightarrow \mathbb{R}$ and $I_{2}$ : $\Psi \rightarrow \mathbb{R}$ be two shape images. Define an invertible and differentiable mapping $\mathbf{f}: \Omega \rightarrow \Psi$ such that $I_{2}(\mathbf{f}(\mathbf{x}))=I_{1}(\mathbf{x})$ (See Figure 1). A deformation-based similarity measure in its most general form can be written as,

$s\left(I_{1}, I_{2}\right)=\min _{\mathbf{f} \in \Gamma} \int_{\Omega} e\left(\mathbf{f}(\mathbf{x}), D \mathbf{f}(\mathbf{x}),(D \mathbf{f}(\mathbf{x}))^{2}, \ldots\right) d \mathbf{x}$

where $e($.$) is the energy functional, D \mathbf{f}$ is the Jacobian of $\mathbf{f}$, and

$$
\begin{array}{r}
\Gamma=\left\{\mathbf{f}: \Omega \rightarrow \Psi \mid I_{2}(\mathbf{f}(\mathbf{x}))=I_{1}(\mathbf{x}) ;\right. \text { and } \\
\operatorname{det}(D \mathbf{f}(\mathbf{x}))>0, \forall \mathbf{x} \in \Omega\},
\end{array}
$$

is a subset of diffeomorphic mappings that register $I_{2}$ to $I_{1}$. The optimization in Eq. (1) is generally non-convex. Hence, the performance of a gradient descent optimization scheme highly depends on the initial guess, $\mathbf{f}_{0}$. Thus, before describing our proposed energy function we describe a method for finding a smooth initial diffeomorphic mapping.

\subsection{Initial mapping}

Input images $I_{1}$ and $I_{2}$ are first centered and rotated to have their major axis aligned (i.e. parallel to the y-axis or any predetermined axis). Then, in order to find an initial smooth diffeomorphic mapping, $\mathrm{f}_{0}: \Omega \rightarrow \Psi$, we pose the problem as a penalized mean squared error (MSE) problem and write,

$$
\begin{array}{r}
\mathbf{f}_{0}=\operatorname{argmin}_{\mathbf{f}} \frac{1}{2} \int_{\Omega}\left(I_{2}(\mathbf{f}(\mathbf{x}))-I_{1}(\mathbf{x})\right)^{2} d \mathbf{x}+ \\
\lambda_{0} \int_{\Omega}\left(\operatorname{det}(D \mathbf{f}(\mathbf{x}))+\frac{1}{\operatorname{det}(D \mathbf{f}(\mathbf{x}))}\right) d \mathbf{x}
\end{array}
$$

where $\lambda_{0}$ is the penalization coefficient. We start the optimization of (2) from the identity mapping. The energy barrier at $\operatorname{det}(D \mathbf{f}(x))=0$ prevents the determinant of the Jacobian from becoming negative (when appropriately small steps are taken in the gradient descent scheme). Thus this registration framework guarantees that a diffeomorphism is obtained. In addition, the penalizing term prevents $\operatorname{det}(D \mathbf{f}(\mathbf{x}))$ from becoming too large or too small.

To solve the optimization problem in Eq. (2) we first decompose $\mathbf{f}$ as, $f(\mathbf{x})=\mathbf{x}+\mathbf{u}(\mathbf{x})$, where $\mathbf{u}(\mathbf{x}): \Omega \rightarrow \Psi$ is the displacement function and represents the amount of deviation from an identity map. Next, we enforce smoothness on the displacement function $\mathbf{u}(\mathbf{x})=[u 1(\mathbf{x}), u 2(\mathbf{x})]^{T}$ by following the same approach as Noblet et al. [12] and representing it as a linear combination of smooth basis $\phi: \Omega \rightarrow \mathbb{R}$ on the image grid points $\mathbf{x}_{i}$.

$$
\left\{\begin{array}{l}
u 1(\mathbf{x})=\sum_{i} \alpha_{i} \phi\left(\mathbf{x}-\mathbf{x}_{i}\right) \\
u 2(\mathbf{x})=\sum_{j} \beta_{j} \phi\left(\mathbf{x}-\mathbf{x}_{j}\right)
\end{array}\right.
$$

Finally, a multi-resolution gradient descent scheme, with respect to $\alpha_{i}^{\prime} \mathrm{s}$ and $\beta_{j}^{\prime} \mathrm{s}$, is used to solve the optimization in Eq. (2). The optimization starts with the identity mapping, $\mathbf{f}(\mathbf{x})=\mathbf{x}$, at the coarsest resolution and updates $\mathbf{f}$, via $\alpha_{i}^{\prime} \mathbf{s}$ and $\beta_{j}$ 's.

We note that, the decomposition of $\mathbf{u}$ in Eq. (3) also simplifies the numerical implementation of our algorithm; as it enables the gradient descent updates for $\alpha_{i}^{\prime}$ s and $\beta_{j}^{\prime}$ s to be expressed in convolution form and implemented using fast Fourier transforms (FFT).

\subsection{Energy terms}

To define a physically meaningful deformation-based metric, we borrow ideas from continuum mechanics. Having deformation $\mathbf{f}$ with associated displacement field $\mathbf{u}$ and displacement Jacobian $D \mathbf{u}$ the strain rate tensor, $E$, is defined as, $E=\frac{1}{2}\left(D \mathbf{u}+D \mathbf{u}^{T}\right)$ [13]. Using $E$ and $\mathbf{f}$, we propose four energy terms, namely viscous friction rate, volume change rate, total mass transport, and compression terms, to capture the differences among deformations. And, the overall energy function, $e($.$) , is defined to be a weighted combination of$ these terms. The viscous friction rate term, $T(\mathbf{f})$, is a measure of strain and is defined as,

$$
\begin{aligned}
& T(\mathbf{f})=4 \int_{\Omega} \operatorname{tr}\left(E(\mathbf{x})^{2}\right) d \mathbf{x} \\
&=4 \int_{\Omega}\left(2 \operatorname{tr}\left(D \mathbf{f}^{2}(\mathbf{x})\right)+2 \operatorname{tr}\left(D \mathbf{f}(\mathbf{x}) D \mathbf{f}^{T}(\mathbf{x})\right)\right. \\
&-8 \operatorname{tr}(D \mathbf{f}(\mathbf{x}))+4 n) d \mathbf{x}
\end{aligned}
$$

where $n$ is the image dimensionality ( $n=2$ or 3 ). The next energy term is the volume change rate, $V(\mathbf{f})$, which is also a measure of strain and is defined as,

$$
\begin{aligned}
V(\mathbf{f}) & =4 \int_{\Omega} \operatorname{tr}(E(\mathbf{x}))^{2} d \mathbf{x} \\
& =4 \int_{\Omega}\left(\operatorname{tr}(D \mathbf{f}(\mathbf{x}))^{2}-2 n \operatorname{tr}(D \mathbf{f}(\mathbf{x}))+n^{2}\right) d \mathbf{x}(5)
\end{aligned}
$$

The third energy term we used penalizes mass transport:

$$
W(\mathbf{f})=\int_{\Omega}|\mathbf{f}(\mathbf{x})-\mathbf{x}|_{2}^{2} I_{1}(\mathbf{x}) d \mathbf{x} .
$$

Minimizing this term alone would give the Wasserstein mass transport distance [14]. This term enables us to measure the amount of mass redistribution in a deformation (e.g. distribution of a certain protein in a cell). Finally, we define the last term, $C(\mathbf{f})$, to represent compression and expansion,

$$
C(\mathbf{f})=\int_{\Omega}\left(\operatorname{det}(D \mathbf{f}(\mathbf{x}))+\frac{1}{\operatorname{det}(D \mathbf{f}(\mathbf{x}))}-2\right)^{2} d \mathbf{x} .
$$

Note that, above energy terms (Eqs. (4)-(7)) are nonsymmetric. Hence the energy function, $e($.$) , is not sym-$ metric, which means that $s\left(I_{1}, I_{2}\right) \neq s\left(I_{2}, I_{1}\right)$. To overcome this issue and provide a symmetric measure we symmetrize the energy terms in a similar fashion as introduced in [15]. 


\subsection{Symmetrization of the energy terms}

Let $\mathbf{g}: \Psi \rightarrow \Omega$ be the inverse of the diffeomorphic map $\mathbf{f}$. We define the symmetric energy terms to be,

$$
\left\{\begin{array}{l}
T_{\text {sym }}(\mathbf{f}, \mathbf{g})=T(\mathbf{f})+T(\mathbf{g}) \\
V_{\text {sym }}(\mathbf{f}, \mathbf{g})=V(\mathbf{f})+V(\mathbf{g}) \\
W_{\text {sym }}(\mathbf{f}, \mathbf{g})=W(\mathbf{f})+W(\mathbf{g}) \\
C_{\text {sym }}(\mathbf{f}, \mathbf{g})=C(\mathbf{f})+C(\mathbf{g})
\end{array}\right.
$$

In order to avoid the calculation of the inverse map, we use the following identities of linear algebra to rewrite the terms corresponding to $\mathrm{g}$ with respect to $\mathbf{f}$. Let $A \in \mathbb{R}^{n \times n}$ be an invertible matrix and $B=A^{-1}$ its inverse. Then,

$$
\left\{\begin{array}{l}
\operatorname{tr}(B)=\frac{\sigma_{n-1}(A)}{\operatorname{det}(A)} \\
\operatorname{tr}\left((B-I)^{2}\right)=\frac{\sigma_{n-1}\left(A^{2}\right)}{(\operatorname{det}(A))^{2}}-2 \frac{\sigma_{n-1}(A)}{\operatorname{det}(A)}+n
\end{array}\right.
$$

where $\sigma_{k}(A)$ is the symmetric polynomial of the eigenvalues of $A$ and is defined as,

$$
\sigma_{k}(A)=\sum_{1 \leq i_{1}<\ldots<i_{k} \leq n} \prod_{j=1}^{k} \lambda_{i_{j}}
$$

where $\lambda_{i_{j}}$ is the $i_{j}$ 'th eigenvalue of $A$. Note that, $\sigma_{1}(A)=$ $\operatorname{tr}(A)$ and $\sigma_{n}(A)=\operatorname{det}(A)$. Hence, for 2D images $(n=2)$ the first energy term for the inverse map, $T(\mathbf{g})$ is,

$$
\begin{aligned}
T(\mathbf{g})= & 4 \int_{\Psi}\left(2 \operatorname{tr}\left(D \mathbf{g}^{2}(\mathbf{y})\right)+2 \operatorname{tr}\left(D \mathbf{g}(\mathbf{y}) D \mathbf{g}^{T}(\mathbf{y})\right)-\right. \\
8 \operatorname{tr}(D \mathbf{g}(\mathbf{y}))+8) d \mathbf{y} & \\
= & 4 \int_{\Omega}\left(2 \frac{\operatorname{tr}\left(D \mathbf{f}^{2}(\mathbf{x})\right)}{\operatorname{det}(D \mathbf{f}(\mathbf{x}))}+2 \frac{\operatorname{tr}\left(D \mathbf{f}(\mathbf{x}) D \mathbf{f}^{T}(\mathbf{x})\right)}{\operatorname{det}(D \mathbf{f}(\mathbf{x}))}-\right. \\
& 8 \operatorname{tr}(D \mathbf{f}(\mathbf{x}))+8(\operatorname{det}(D \mathbf{f}(\mathbf{x})))) d \mathbf{x}
\end{aligned}
$$

where in the last equation we used the change of variables $\mathbf{y}=\mathbf{f}(\mathbf{x}), d \mathbf{y}=\operatorname{det}(D \mathbf{f}(\mathbf{x})) d \mathbf{x}$, and the linear algebra identities in Eq. 9. Similarly, for volume change we write,

$$
\begin{aligned}
V(\mathbf{g}) & =4 \int_{\Psi}\left((\operatorname{tr}(D \mathbf{g}(\mathbf{y})))^{2}-4 \operatorname{tr}(D \mathbf{g}(\mathbf{y}))+4\right) d \mathbf{y} \\
& =4 \int_{\Omega}\left(\frac{(\operatorname{tr}(D \mathbf{f}(\mathbf{x})))^{2}}{\operatorname{det}(D \mathbf{f}(\mathbf{x}))}-4 \operatorname{tr}(D \mathbf{f}(\mathbf{x}))\right. \\
& +4 \operatorname{det}(D \mathbf{f}(\mathbf{x}))) d \mathbf{x} .
\end{aligned}
$$

And for the transport term we have,

$$
\begin{aligned}
W(\mathbf{g}) & =\int_{\Psi}\left(|\mathbf{g}(\mathbf{y})-\mathbf{y}|^{2} I_{2}(\mathbf{y})\right) d \mathbf{y} \\
& =\int_{\Omega}\left(|\mathbf{f}(\mathbf{x})-\mathbf{x}|^{2} I_{1}(\mathbf{x}) \operatorname{det}(D \mathbf{f}(\mathbf{x}))\right) d \mathbf{x} .
\end{aligned}
$$

Finally, for the compression term we write,

$$
\begin{aligned}
& C(\mathbf{g})=\int_{\Psi}\left(\operatorname{det}(D \mathbf{g}(\mathbf{y}))+\frac{1}{\operatorname{det}(D \mathbf{g}(\mathbf{y}))}-2\right) d \mathbf{y} \\
&=\int_{\Omega}\left(\left(\operatorname{det}(D \mathbf{f}(\mathbf{x}))+\frac{1}{\operatorname{det}(D \mathbf{f}(\mathbf{x}))}-2\right)\right. \\
&\operatorname{det}(D \mathbf{f}(\mathbf{x}))) d \mathbf{x}
\end{aligned}
$$

In this manner, the symmetric energy terms can be solely written as a function of the forward transform, $\mathbf{f}$, and without the need for calculation of the inverse mapping.

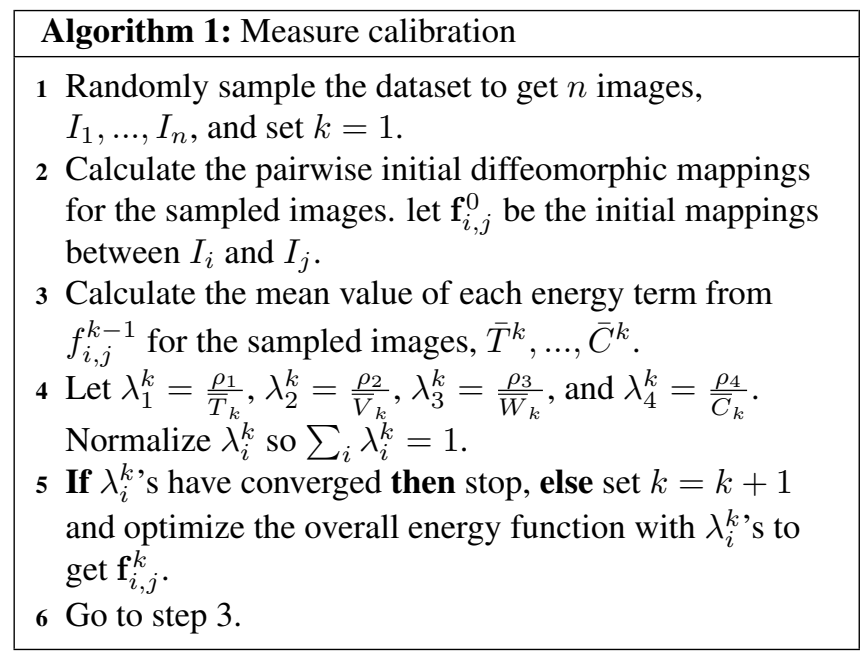

\subsection{Symmetric deformation-based similarity measure}

For two shape images $I_{1}$ and $I_{2}$, and using the symmetrized energy terms, we propose the following distance,

$$
\begin{aligned}
s\left(I_{1}, I_{2}\right)=\min _{\mathbf{f}} & \lambda_{1} T_{\text {sym }}(\mathbf{f})+\lambda_{2} V_{\text {sym }}(\mathbf{f}) \\
& +\lambda_{3} W_{\text {sym }}(\mathbf{f})+\lambda_{4} C_{\text {sym }}(\mathbf{f}) \\
\text { s.t. } & I_{2}(\mathbf{f}(\mathbf{x}))=I_{1}(\mathbf{x}), \forall \mathbf{x} \in \Omega
\end{aligned}
$$

where $\lambda_{i}^{\prime}$ s are the mixing coefficients of energy terms. Above optimization is then solved using the method of Lagrange multipliers together with a gradient descent scheme which starts from the pre-calculated initial diffeomorphic mapping. We note that since our method enforces local injectivity in a connected domain it is also globally injective.

Each energy term quantifies specific characteristic of the deformation mapping. This enables the scientists to provide the relative importance of each term that matches their specific experiment. For instance, when the goal of an experiment is to model the distribution of a certain protein in the cell, the mixing coefficient of the transport term, $\lambda_{3}$, should be set to a relatively higher value to express the importance of this term.

\subsection{Measure calibration}

The mixing coefficients, $\lambda_{i}^{\prime} \mathrm{s}$ in Eq. (15), should be tuned to incorporate the relative importance of each term provided by the user, and also normalize the order of magnitude of different terms. To do so, we devised an automatic method, which takes as input the shape dataset and the relevant importance of terms $\rho_{i}$ with $\sum_{i} \rho_{i}=1$ and determines $\lambda_{i}^{\prime}$ s iteratively. Algorithm 1 shows the measure calibration steps.

\section{EXPERIMENTAL RESULTS}

To test our proposed method we used 2D fluorescence microscope images of HeLa cells [5] provided by Murphy Lab, 


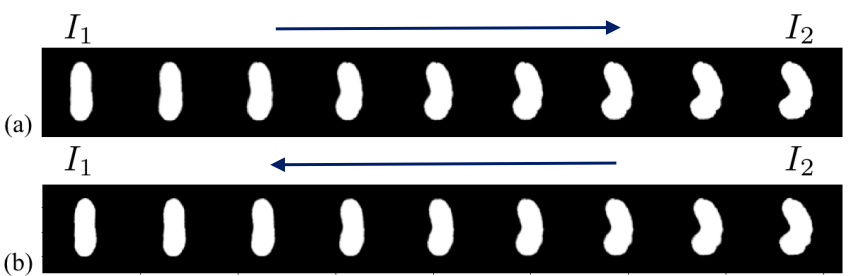

Fig. 2. The deformation interpolation calculated from $I_{1}$ to $I_{2}$ (a) and from $I_{2}$ to $I_{1}$ (b) using our proposed method. The symmetric nature of SDBM is self evident and the relative error between $s\left(I_{1}, I_{2}\right)$ and $s\left(I_{2}, I_{1}\right)$ is $0.6 \%$.

Carnegie Mellon University, Pittsburgh. The dataset contains images from 10 different channels of $70 \mathrm{HeLa}$ cells. We used the 'nuclei' channel to generate binary images of cell nuclei shapes, as it plays an important role in cancer prognosis in a wide variety of cancers. The pairwise similarity of these shape images are then calculated using our proposed method, LDDMM, and the standard Euclidean distance.

For our method, the relative importance of the energy terms are set to $\rho_{i}=0.25$, for $i=1, \ldots, 4$. The number of sampled images, $n$, used for tuning the mixing coefficients is set to $10 \%$ of the size of the dataset and the coefficients are automatically tuned as described in Algorithm 1. The pairwise similarity/distances in the dataset are then calculated by minimizing the energy function in Eq. (15). Figure 2 shows interpolants calculated from two sample images, $I_{1}$ and $I_{2}$ for our method. Let $\mathbf{f}_{12}$ and $\mathbf{f}_{21}$ be the optimal mappings that map $I_{2}$ to $I_{1}$ and $I_{1}$ to $I_{2}$, respectively. The geodesics shown in Figure 2 are calculated from, $I_{1}\left((1-\alpha) \mathbf{x}+\alpha \mathbf{f}_{12}(\mathbf{x})\right)$ and $I_{2}\left((1-\alpha) \mathbf{x}+\alpha \mathbf{f}_{21}(\mathbf{x})\right)$, for $\alpha \in[0,1]$, respectively.

For the LDDMM distance, we used the implementation provided by Rohde et al. [3] and used the same parameters as suggested in [3]. In addition, the Euclidean distance is calculated as the square root of the sum of the squared pixel differences between images.

Given the shape images $I_{1}, \ldots, I_{N}$, we define $A$ to be the pairwise distance matrix for the dataset, for which $A_{i, j}=$ $s\left(I_{i}, I_{j}\right)$, and $s(.,$.$) is the determined similarity measure. Us-$ ing MDS we find a set of coordinates $\mathbf{w}_{k} \in \mathbb{R}^{M}$ to embed the images in a $M$-dimensional vector space in which the pairwise Euclidean distances closely resemble the similarity measure calculated from $s(.,$.$) in the original space. The es-$ timated distance matrix in the low-dimensional embedding is $\tilde{A}_{i, j}=\left\|\mathbf{w}_{i}-\mathbf{w}_{j}\right\|$.

The intrinsic dimensionality of the variations in a dataset can be estimated from the decrease in the residual variance [16, 3] between matrices $A$ and $\tilde{A}$. Similar to [16] and [3], we let the residual variance to be $1-R^{2}(A, \tilde{A})$, where $R(.,$. denotes the standard correlation coefficient between two matrices. A good similarity measure should be capable of capturing the intrinsic dimensionality of a dataset. However, the intrinsic dimensionality of the data is not known, hence, a good measure can be characterized as the one that provides the least

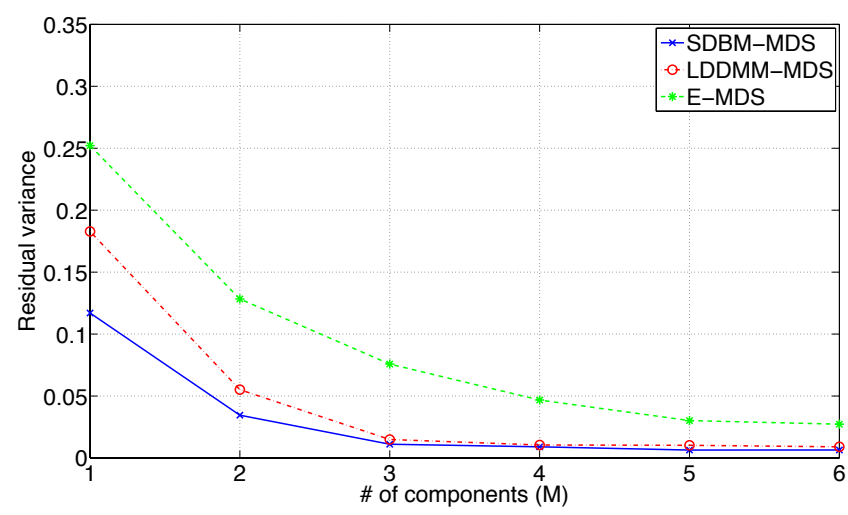

Fig. 3. Residual variance between original and reconstructed using Euclidean distance MDS (E-MDS), LDDMM-MDS, and our proposed distance MDS (SDBM-MDS). The curves show the rate by which the residual variance decreases as more components are included in the dimensionality reduction process.

residual variance in a low-dimensional Euclidean embedding. Figure 3 shows the measured residual variance as a function of the dimensionality of the low-dimensional Euclidean embedding $(M)$ for our proposed method (SDBM-MDS), LDDMM MDS, and Euclidean MDS (E-MDS). It can be seen that SDBM-MDS provides the lowest residual variance.

\section{SUMMARY AND DISCUSSION}

In this paper, we introduced a symmetric deformation-based similarity measure for cell shapes with application to statistical cell modeling. The proposed similarity measure incorporates several physically meaningful symmetric energy terms that can be used to capture and model differences in a wide variety of cell shape analysis problems. We compared our proposed method with LDDMM and the standard Euclidean distance and showed that SDBM is capable of modeling the variations in the dataset with fewer parameters and with more fidelity. In addition, the gradient descent steps in SDBM can be written in convolution form, which enables a fast numerical implementation of the problem that scales up linearly for higher dimensional shape analysis. Although the proposed approach does not provide a true metric, but it is a physically meaningful similarity measure, and a metric can be defined on the corresponding similarity graph of the data.

\section{ACKNOWLEDGEMENT}

Authors wish to express their thanks to Prof. Robert Murphy (CMU) for discussions on the topic. This work was partially supported by NIH (grant GM-103712) and the John and Claire Bertucci Graduate Fellowship. Authors acknowledge the support by NSF (grant CCF-1421502). DS is grateful to NSF (grant DMS-1211760) for its support. 


\section{REFERENCES}

[1] John Ashburner, Chloe Hutton, Richard Frackowiak, Ingrid Johnsrude, Cathy Price, Karl Friston, et al., "Identifying global anatomical differences: deformation-based morphometry," Human brain mapping, vol. 6, no. 5-6, pp. 348-357, 1998.

[2] Michael I Miller, "Computational anatomy: shape, growth, and atrophy comparison via diffeomorphisms," NeuroImage, vol. 23, pp. S19-S33, 2004.

[3] Gustavo K Rohde, Alexandre JS Ribeiro, Kris N Dahl, and Robert F Murphy, "Deformation-based nuclear morphometry: Capturing nuclear shape variation in hela cells," Cytometry Part A, vol. 73, no. 4, pp. 341-350, 2008.

[4] Qian Xie, Sebastian Kurtek, Gary E Christensen, Zhaohua Ding, Eric Klassen, and Anuj Srivastava, "A novel framework for metric-based image registration," in Biomedical Image Registration, pp. 276-285. Springer, 2012.

[5] Michael V Boland and Robert F Murphy, "A neural network classifier capable of recognizing the patterns of all major subcellular structures in fluorescence microscope images of hela cells," Bioinformatics, vol. 17, no. 12, pp. 1213-1223, 2001.

[6] Z Pincus and JA Theriot, "Comparison of quantitative methods for cell-shape analysis," Journal of microscopy, vol. 227, no. 2, pp. 140-156, 2007.

[7] Mingqiang Yang, Kidiyo Kpalma, and Joseph Ronsin, "A survey of shape feature extraction techniques," Pattern recognition, pp. 43-90, 2008.

[8] Timothy F Cootes, Christopher J Taylor, David H Cooper, and Jim Graham, "Active shape models-their training and application," Computer vision and image understanding, vol. 61, no. 1, pp. 38-59, 1995.

[9] M Faisal Beg, Michael I Miller, Alain Trouvé, and Laurent Younes, "Computing large deformation metric mappings via geodesic flows of diffeomorphisms," International journal of computer vision, vol. 61, no. 2, pp. 139-157, 2005.

[10] Joseph B Kruskal, "Multidimensional scaling by optimizing goodness of fit to a nonmetric hypothesis," Psychometrika, vol. 29, no. 1, pp. 1-27, 1964.

[11] Monica Hernandez, Matias N Bossa, and Salvador Olmos, "Registration of anatomical images using paths of diffeomorphisms parameterized with stationary vector field flows," International Journal of Computer Vision, vol. 85, no. 3, pp. 291-306, 2009.
[12] Vincent Noblet, Christian Heinrich, Fabrice Heitz, and J-P Armspach, "Retrospective evaluation of a topology preserving non-rigid registration method," Medical image analysis, vol. 10, no. 3, pp. 366-384, 2006.

[13] Oscar Gonzalez and Andrew M Stuart, A first course in continuum mechanics, Cambridge University Press, 2008.

[14] C. Villani, Topics in Optimal Transportation, Graduate Studies in Mathematics. American Mathematical Society, 2003.

[15] Pascal Cachier and David Rey, "Symmetrization of the non-rigid registration problem using inversion-invariant energies: application to multiple sclerosis," in Medical Image Computing and Computer-Assisted InterventionMICCAI 2000. Springer, 2000, pp. 472-481.

[16] Joshua B Tenenbaum, Vin De Silva, and John C Langford, "A global geometric framework for nonlinear dimensionality reduction," Science, vol. 290, no. 5500, pp. 2319-2323, 2000. 